\title{
Dealing with Stochasticity in Wood Remanufacturing Operations Planning
}

\author{
Rezvan Rafiei \\ Business School, Université de Sherbrooke, Sherbrooke, Canada, \\ 2500, boulevard de l'Université Sherbrooke, Québec, QC, J1K 2R1, Canada. \\ E-mail: rezvan.rafiei@usherbrooke.ca; rezvan.rafiei@gmail.com \\ Mustapha Nourelfath \\ Department of Mechanical Engineering, Université Laval, Quebec, Canada, \\ Local 3344, Pavillon Adrien-Pouliot, Université Laval, Québec, QC, Canada. \\ G1V 0A6. Telephone: (418) 656-2131\#12355. Fax: (418) 656-7415, \\ E-mail: Mustapha.Nourelfath@gmc.ulaval.ca \\ Luis Antonio De Santa-Eulalia \\ Business School, Université de Sherbrooke, Sherbrooke, Canada, \\ 2500, boulevard de l'Université Sherbrooke, Québec, QC, J1K 2R1, Canada. \\ Telephone: (819) 821-8000\#65042. \\ Corresponding author: 1.santa-eulalia@usherbrooke.ca
}

(Received March 19, 2020; Accepted August 28, 2020)

\begin{abstract}
The planning process of wood remanufacturing operations encompasses challenging characteristic, including divergent co-production (one log tree may produce several different products), alternative processes (different receipts exist to produce the same products), short order cycle, dynamic market behaviour (with highly varying demand) and imperfect raw materials (due to its biological nature, the yield vary considerably). To deal with this complexity, in this paper random demand is modeled as scenario tree and three new predictive multi-stage stochastic programming models are developed with multiple objective functions. After implementing them employing datasets from a wood remanufacturing partner in Canada, the proposed models are compared to a reactive re-planning approach. The obtained results indicate that the new models exhibit higher quality solutions in comparison with their corresponding deterministic two-stage models. We also determine the number of stages for which the multi-stage programs provide better planning than the re-planning approach.
\end{abstract}

Keywords- Multi-stage stochastic programming, Production planning, Demand uncertainty, Co-production, Wood remanufacturing sector.

\section{Introduction}

In the wood remanufacturing sector price-based competition, sawmills are primary processing plants seeking to develop value-added products, which are forwarded to secondary and tertiary plants called wood remanufacturing mills. In this context, low value-added lumbers (usually with quality issues) are transformed into several high-value end-products (e.g. components for furniture, roofing, etc.).

Some basic features of this industry sector make its production planning quite challenging, among them it is important to mention the following ones. First of all, remanufacturing mills transform different finished products in the same production run using an intrinsic divergent co-production approach. In addition, one item can be manufactured through alternative processes (called recipes), using substitute raw materials (i.e., lumber) that, in turn, output different co-products. Alternative 
processes differ in terms of co-products yield, required inputs, production lead-times and also production costs. Moreover, manufacturing planning has to deal with a dynamic market, encompassing a large product portfolio with changing demands and short-term delivery lead-times. Many unpredictable orders are received at the same time and must be filled in a brief period. The dominant marketing practices in remanufacturing sector ask for a make-to-order production planning, in which serval products are manufactured according to clients orders, but forcibly other less important co-products are produced and later stocked. Furthermore, the raw materials are imperfect, i.e. transferred lumber from sawmills has some visual features that are critical in remanufacturing plants. In fact, different recipes are defined for a specific lumber according to different unusable areas (imperfect spots) spread throughout lumber. Most of these imperfections are in the form of knots, varying location and form, typical in biological material. This issue is especially relevant in the studied industry sector.

Production planning is not simple in this context, particularly when demand varies considerably. In the literature, first papers dealing with planning approaches for this industrial field were Rafiei et al. (Rafiei et al., 2014; Rafiei et al., 2015; Rafiei et al., 2016); in which a series of reactive planning approaches is developed and evaluated, making use of complete re-planning approach following a periodic policy and rolling horizons. This kind of reactive planning approaches can provide good results when demands are unpredictable. However, but combined with local and realtime decisions, with small order cycle-times, planners have to deal with short re-planning intervals. As a result, one sees an increase in the number of revisions that a plan undergoes during execution, affecting plan stability and nervousness (Vieria et al., 2003), which may not be suitable in the real world. In addition, these reactive planning approaches may not be efficient in conditions with more stable demand (i.e. when a more mature product family, with consistent historical data), which can be the case in this sector in several situations. Thus, the existing approaches miss some important research opportunities.

To contribute reducing this research gap, the presence of both high-level re-planning frequency and an important quantity of mature products together in the same supply chain has motivated us to develop and test a series of novel stochastic models, created as a predictive approach with finite distributions for the stochastic demand parameter. In this context, considering that the demand probability distribution is known, this paper develops a predictive (rather than reactive) approach based on multi-stage stochastic programming. The new proposed models are submitted to a comparative analysis, benchmarking the existing reactive approach developed in Rafiei et al. (Rafiei et al., 2014; Rafiei et al., 2015; Rafiei et al., 2016). An important result consists in determining the number of stages for which the multi-stage programs provide better planning than the re-planning approach. This is the first time this kind of problem has been modelled through stochastic programming in the wood remanufacturing sector, and compared to a reactive planning approach. This work thus extends the previous research and contributes by providing the wood remanufacturing industry with both reactive and predictive planning systems necessary to deal with different market conditions. To model a realistic production planning set, a real case study in Canada is considered as a proof-of-concept case study.

This manuscript is organized into four sections: Section 2 presents a literature review and it specifies the paper's contribution. The proposed planning approach is presented in Section 4 together with the proof-of-concept case study. Section 4 discusses computational results and Section 5 presents the paper's conclusions. 
International Journal of Mathematical, Engineering and Management Sciences

Vol. 6 No. 2, 522-540, 2021

https://doi.org/10.33889/IJMEMS.2021.6.2.032

\section{Literature Review}

Basically, two research streams are connected to the present work: stochastic programming in production planning problems, which is complementary to the wood remanufacturing industry planning problems.

First of all, stochastic programming approaches have been employed in production planning problems since long ago, in which decision systems comprising stochastic processes are encapsulated in a finite number of stages with uncertainty evolving over time. Due to computational burden to solve these types of problems, a series of stochastic programing algorithms has been created for solving industry-scale problems (Ruszczynski and Shapiro, 2003). For most of them, a two-stage model is considered. Examples of works dealing with demand uncertainty include design batch plants (Engell et al., 2004; Chu and You, 2013), supply chain planning (Gupta and Maranas, 2003), semiconductor capacity planning (Swaminathan, 2000; Rao et al., 2004) and forestry products planning (Kazemi et al., 2011; Shabani et al., 2014). By allowing the decisions to be revised at any time during the stage upon the realized uncertainty, an extension of the two-stage paradigm to the multi-stage stochastic programming occurs (Huang and Ahmed, 2009). In such problems, a scenario tree describes the uncertainty and an objective function is defined to indicate the related risks in the decision-making process. The literature in multi-stage stochastic programming to deal with uncertain demand focus diverse areas, including: capacity planning (Karabuk and Wu, 2003; Pimentel et al., 2013), lot-sizing (Brandimarte, 2006; Huang and Ahmed, 2009) and forestry production planning (Kazemi et al., 2010).

Secondly, the stream related to the wood remanufacturing industry is also evolving considerably. Generic models for remanufacturing were proposed: Farrell and Maness (2005) came up with a decision-support system for production planning in secondary (remanufacturing) mills using generic linear programming models, tested in a real case located in British Columbia (Canada). Additionally, some researchers in this industry incorporated lean manufacturing (Pirraglia et al., 2009), i.e. instead of trying to predict the future, some lean manufacturing principles are employed to establish inventory levels, disregarding replenishment times and customer demand variations. Despite the fact that secondary wood processing industries have brought massive social and economic benefits since the early 1980s (Kozak et al., 2003), companies in this sector still face critical challenges in production planning under dynamic demand. More recently, some studies consider production planning problems in the wood remanufacturing with this dynamic aspect in mind (Rafiei et al., 2014; Rafiei et al., 2015; Rafiei et al., 2016).

This literature review indicates that, despite the existence of several works in the field of production planning under uncertain demand through stochastic programming, it can still be considered an developing field comprising diverse uncluttered research questions (Rönnqvist et al., 2015). In addition, some works have studied optimization in production planning in the forest products sector (e.g., D'Amours et al., 2008) and stochastic programming is used (e.g., Kazemi et al., 2011; Shabani et al., 2014). However, the remanufacturing industry holds some characteristics (already discusses in Section 1) that distinguish it from other manufacturing operations, making this a complex sector to manage. It is still missing an application of stochastic programming explicitly considering the nature of the wood remanufacturing industry in the literature.

Thus, the present study develops three multi-stage stochastic programming models wherein demand uncertainty evolves with time according to a stochastic process. Each optimization model has a different objective function, namely the cost, the marginal contribution, and the profit. These 
stochastic models aim to provide production plans that encompass the following chatacteristcs: they have practical implementation viability, they are able to manage different demand scenarios, allowing for a future full-recourse action. Predictive in nature, the proposed multi-stage stochastic models are compared to the reactive approach. As already indicated in the introduction, this is the first time this kind of problem has been modeled through stochastic programming in the wood remanufacturing sector. The proposed predictive multi-stage stochastic models are compared to a reactive rolling-horizon based re-planning method, aiming to identify the number of stages for which the multi-stage programs provide better planning than the re-planning approach. This allows for an evaluation of the gain of using predictive stochastic programming models instead of a reactive planning approach that increases the system nervousness and instability. Another important contribution of the paper is that the proposed approach is applied for a real case study of wood remanufacturing production planning, which is presented next.

\section{Contribution: Proof-of-Concept and Mathematical Models}

We consider a softwood remanufacturing mill located in Quebec, Canada. The case under study transforms lumber into bed frame components. It is the same case study of Rafiei et al. (Rafiei et al., 2014; Rafiei et al., 2015; Rafiei et al., 2016), which will be later used as a benchmarking reference for the new stochastic models developed herein for the same industry sector and for a proof-of-concept and comparative analysis in Section 4.

In this case, production involves lumber sorting, drying and remanufacturing. In a co-production system with alternative processes or recipes, each lumber (as raw materials) may have more than one recipe, producing different sets of co-product. We invite the reader to consult Rafiei et al. (2014) for a detailed explanation of the case employed as a reference here.

In the next subsections, we first introduce the deterministic models. Next, we present our methodology in modeling the uncertain demand before providing our new stochastic programming formulations of the proposed models.

\subsection{Deterministic Models}

Consider a production unit with $T$ periods as a planning horizon, in which $t$ refers to periods $t=1, \ldots, T$. Supply of raw materials is considered unlimited. We also consider a single machine with negligible setup time.

\subsubsection{Notations}

The notations used are:

$$
\begin{array}{ll}
\text { Sets } & \\
P^{\text {consumed }} & \text { Products } p \text { that can be consumed } \\
P^{\text {produced }} & \text { Products } p \text { that can be produced } \\
R & \text { Set of recipes } r \text { (an alternative process) } \\
\text { Parameters } & \\
c_{r t} & \text { Production costs associated with using recipe } r \text { in period } t \\
i n v_{p t} & \text { Inventory holding cost per unit of products } p \in P^{\text {produced }} \text { in } \\
b o_{p t} & \text { Backorder cost per unit of product } p \in P^{\text {produced }} \text { in period } t
\end{array}
$$$$
P \text { Produced } \quad \text { Products } p \text { that can be produced }
$$$$
\text { inv } v_{p t} \quad \text { Inventory holding cost per unit of products } p \in P^{\text {produced }} \text { in period } t
$$ 


$\begin{array}{ll}\delta_{r} & \text { Capacity required for each recipe } r \text { per unit time } \\ c_{t} & \text { Available capacity of machine for period } t \\ i c_{p 0} & \text { The inventory of raw material } p \in P^{\text {consumed }} \text { at the beginning of planning horizon } \\ s_{p t} & \text { Supply of raw material } p \in P^{\text {consumed }} \text { provided at the beginning of period } t \\ i p_{p 0} & \text { The inventory of product } p \in P^{\text {produced }} \text { at the beginning of planning horizon } \\ \phi_{p r} & \text { The units of raw material } p \in P^{\text {consumed }} \text { consumed by recipe } r \\ \rho_{p r} & \text { The quantity of product } p \in P^{\text {produced }} \text { produced by recipe } r \\ d_{p t} & \text { Demand of product } p \in P^{\text {produced }} \text { to be delivered by the end of period } t \\ m c_{r} & \text { Marginal contribution of recipe } r \text { that is the marginal profit of all products to be } \\ r_{p r} & \text { produced simultaneously using recipe } r \\ D e c i s i o n & \text { Selling price (revenue) per unit of product } p \in P^{\text {produced }} \text { according to recipe } r \\ X_{r t} & \text { Number of times each recipe } r \text { should be run in period } t \\ I P_{p t} & \text { Inventory size of product } p \in P^{\text {produced }} \text { by the end of period } t \\ B O_{p t} & \text { Backorder size of product } p \in P^{\text {produced }} \text { by the end of period } t \\ I C_{p t} & \text { Inventory size of raw material } p \in P^{\text {consumed }} \text { by the end of period } t \\ F_{p t} & \text { Quantity sold of product } p \in P^{\text {produced }} \text { by the end of period } t\end{array}$

\subsubsection{Models}

We consider several alternative optimization models as deterministic models. Each optimization model has a different objective function, namely the cost, the marginal contribution, and the profit. In the following, first the objective functions are defined and then the constraints are presented.

\section{A. Objective Functions}

Model 1: Cost minimization

The objective function of Model 1 (Equation 1) minimizes total production costs, inventory holding costs and backorder costs for all products.

$$
\text { Minimize } \sum_{t}^{T} \sum_{r \in R} c_{r t} X_{r t}+\sum_{t}^{T} \sum_{p \in P^{p r o d u c e d}}\left(i n v_{p t} I P_{p t}+b o_{p t} B O_{p t}\right)
$$

Model 2: Marginal Contribution Maximization

The second model consists in maximizing the "marginal contribution", which is calculated as the prices of end products minus their total variable costs of production and consumed raw materials. Considering marginal contribution is a common optimization practice because of the co-production system in such mills. The objective function of Model 2 is: 


$$
\text { Maximize } \sum_{t}^{T} \sum_{r \in R} m c_{r} X_{r t}-\sum_{t}^{T} \sum_{p \in P^{p r o d u c e d}}\left(i n v_{p t} I P_{p t}+b o_{p t} B O_{p t}\right)
$$

Model 3: Profit Maximization

The third model aims to maximize "profit" (i.e. total revenue minus total costs).

$$
\begin{aligned}
& \text { Maximize } \sum_{t}^{T} \sum_{r \in R} \sum_{p \in P^{\text {produced }}} r_{p r} F_{p t}- \\
& \left(\sum_{t}^{T} \sum_{r \in R} c_{r t} X_{r t}+\sum_{t}^{T} \sum_{p \in P^{\text {produced }}}\left(i n v_{p t} I P_{p t}+b o_{p t} B O_{p t}\right)\right)
\end{aligned}
$$

\section{B. Constraints}

Constraints of Models 1 and 2 are Constraints (4)-(8) and Constraints (12)-(14). However, Model 3 requires Constraints (4)-(6) and Constraints (9)-(15).

$$
\begin{aligned}
& \sum_{\forall r \in R} \delta_{r} X_{r t} \leq c_{t} \quad \forall t=1, \ldots, T \\
& I C_{P 1}=i c_{p 0}+s_{p 1}-\sum_{\forall r \in R} \phi_{p r} X_{r 1} \quad \forall p \in p^{\text {consumed }}, t=1 \\
& I C_{p t}=I C_{p t-1}+s_{p t}-\sum_{\forall r \in R} \phi_{p r} X_{r t} \quad \forall p \in p^{\text {consumed }}, t=2, \ldots, T \\
& I P_{p 1}-B O_{P 1}=i p_{p 0}+\sum_{\forall r \in R} \rho_{p r} X_{r 1}-d_{p 1} \quad \forall p \in P^{\text {produced }}, \quad t=1 \\
& I P_{p t}-B O_{p t}=I P_{p t-1}-B O_{p t-1}+\sum_{\forall r \in R} \rho_{p r} X_{r t}-d_{p t} \quad \forall p \in p^{\text {produced }}, \quad t=2, \ldots, T \\
& I P_{p 1}-B O_{P 1}=i p_{p 0}+\sum_{\forall r \in R} \rho_{p r} X_{r 1}-F_{p 1} \quad \forall p \in P^{\text {produced }}, \quad t=1 \\
& I P_{p t}-B O_{p t}=I P_{p t-1}-B O_{p t-1}+\sum_{\forall r \in R} \rho_{p r} X_{r t}-F_{p t} \quad \forall p \in p^{\text {produced }}, \quad t=2, \ldots, T \\
& F_{p t} \leq d_{p t} \quad \forall p \in P^{\text {produced }}, t=1, \ldots, T \\
& X_{r t} \geq 0 \quad \forall r \in R, \quad t=1, \ldots, T \\
& I C_{p t} \geq 0 \quad \forall p \in P^{\text {consumed }}, t=1, \ldots, T \\
& I P_{p t} \geq 0, B O_{p t} \geq 0 \quad \forall p \in P^{\text {produced }}, t=1, \ldots, T \\
& F_{p t} \geq 0 \quad \forall p \in P^{\text {produced }}, t=1, \ldots, T
\end{aligned}
$$

Constraint (4) guarantees that the total production time is not greater than the available time and manufacturing capacity. Both (5) and (6) were created to ensure the total inventory balance for raw materials, i.e. inventory at the end of period $t$ is equal to its inventory in $t$ - 1 , plus raw materials 
$p \in P^{\text {consumed }}$ supplied $\left(s_{p t}\right)$ at the beginning of that period, minus its total utilisation in that period. In addition, constraints (7) and (8) aim to ensure that the sum of inventory (or backorder) of product $p \in P^{\text {produced }}$ at the end of period $t$ is equivalent to its inventory (or backorder) in the period $t-1$, plus the total production of that product in that period, minus the product demand for that period. Constraints (9) and (10) guarantee that the sales quantities should be satisfied by the production as well as the available inventories or backorder. Constraint (11) ensures that sales do not exceed customer demand at each period in Model 3. Finally, Constraints (12)-(15) ensures the decision variables' non-negativity the.

\subsection{Modelling Demand}

A scenario tree represents a common structure showing how uncertainty evolves over time, comprising potential data sequences (Alonso-Ayuso et al., 2003) in a planning horizon divided into time intervals. A stage is related to a time interval where a decision is made, and the length of the time interval might vary from one to a limited number of periods in a stage. The first stage represents the current date. Any tree node corresponding to a time interval represents a different possible state of the system, and arcs indicate the possible transaction from a state in a time interval to a new state in the next time interval, with a given probability of this transition occurring. We use the Gaussian approximation method according to Miller and Rice (1983) to depict the scenario tree. That is, a trinomial scenario tree is composed of three possible demand levels, being High, Average, and Low, representing three possible realizations. The planning horizon is 54 periods organized in 4 stages, which means each one includes 18 periods. This produces a scenario tree with 40 nodes organized into 27 demand scenarios.

\subsection{Multi-Stage Stochastic Programs}

A multi-stage stochastic programming approach is employed to address uncertainty over time (Birge and Louveaux, 2010), given current resources, as well as possible future uncertainties and recourse actions. Uncertainties are modeled through a scenario tree (similar to the one presented in the previous subsection), an objective function captures the risk related to the sequence of decisions to be made and the mathematical problem is solved by way of a large-scale linear program.

Depending on the mathematical formulation for stochastic programming models, it might be necessary to ensure that the decision sequence respects the information structure associated with the scenario tree (Higle, 2005). It means that the model reflects non-anticipativity of the system (or is implementable), in which a decision have to be made in each stage with no knowledge of future realizations of random variables in further stages. Two approaches can enforce non-anticipativity constraints in the multi-stage model, namely split variable formulations and compact formulations (Alfieri and Brandimarte, 2005). In split variable formulations, the non-anticipativity constraints are explicitly imposed through a set of decision variables for each time period and each scenario. In contrast, in compact formulations, decision variables are associated to the nodes of the tree and non-anticipativity is built in a more implicit way. As compact formulations are computationally cheaper than split variable formulations when using standard solvers (Alfieri and Brandimarte, 2005), we employ a compact formulation for this problem. This assures that solutions at one stage does not depend on information not yet available, creating what literature calls full recourse (Alonso-Ayuso et al., 2003). In what follows, we at first present the notations, and then the multistage model. 
International Journal of Mathematical, Engineering and Management Sciences

Vol. 6 No. 2, 522-540, 2021

https://doi.org/10.33889/IJMEMS.2021.6.2.032

\subsubsection{Notations}

Complementing those notations provided in Section 4.1.1, following mathematical notations are employed:
Indices
$\tau \quad$ Scenario tree
$n, n^{\prime} \quad$ Nodes of the scenario tree $n, n^{\prime} \in \tau$
Sets
$\lambda_{n} \quad$ Predecessor of node $n$ in the scenario tree
$\begin{array}{ll}\theta_{n} & \text { Set of time periods corresponding to node } n \text { in the scenario tree (number of periods } \\ \text { in one stage) }\end{array}$
Parameters
$d_{p t}^{n} \quad$ Demand of product $p \in P^{\text {produced }}$ to be delivered by the end of period $t$ at node $n$ of
$\pi_{n} \quad$ Probability of node $n$ of the scenario tree

\section{Decision variables}
$X_{r t}^{n} \quad \begin{aligned} & \text { Control variable - Number of times each recipe } r \text { should be run in period } t \text { at node } n \text { of } \\ & \text { the scenario tree }\end{aligned}$
$I C_{p t}^{n} \quad$ Inventory size of raw material $p \in P^{\text {consumed }}$ by the end of period $t$ at node $n$ of the scenario tree
$I P_{p t}^{n} \quad$ State variable - Inventory size of product $p \in P^{\text {produced }}$ by the end of period $t$ at node $n$ of the scenario tree
$B O_{p t}^{n} \quad$ State variable - Backorder size of product $p \in P^{\text {produced }}$ by the end of period $t$ at node $n$ of the scenario tree
$F_{p t}^{n} \quad$ Quantity sold of product $p \in P^{\text {produced }}$ by the end of period $t$ at node $n$ of the scenario tree

\subsubsection{Model}

\section{A. Objective Functions}

For the three considered alternatives in the deterministic models (Section 4.1.2.), three objective functions are proposed in the multi-stage stochastic models.

Model 1: Expected Cost Minimization

The objective function's first term of Model 1 (Equation 16) calculates the expected production costs, while the second term accounts for the expected inventory holding and backorder costs

$$
\text { Minimize } \sum_{n \in \tau} \pi_{n}\left(\sum_{t \in \theta_{n}}^{T} \sum_{r \in R} c_{r t} X_{r t}^{n}+\sum_{t \in \theta_{n}}^{T} \sum_{p \in P^{\text {produced }}}\left(i n v_{p t} I P_{p t}^{n}+b o_{p t} B O_{p t}^{n}\right)\right)
$$

Model 2: Expected Marginal Contribution Maximization

The first term of the objective function of Model 2 (Equation 17) is the expected marginal contribution for the demand scenario tree and the second one computes the expected inventory holding and backorder costs. 


$$
\text { Maximize } \sum_{n \in \tau} \pi_{n}\left(\sum_{t \in \theta_{n}}^{T} \sum_{r \in R} m c_{r} X_{r t}^{n}-\sum_{t \in \theta_{n}}^{T} \sum_{p \in P^{p r o d u c e d}}\left(i n v_{p t} I P_{p t}^{n}(n)+b o_{p t} B O_{p t}^{n}\right)\right)
$$

Model 3: Expected Profit Maximization

The objective function of Model 3 (Equation 18) includes the expected revenue and the expected production, inventory holding and backorder costs.

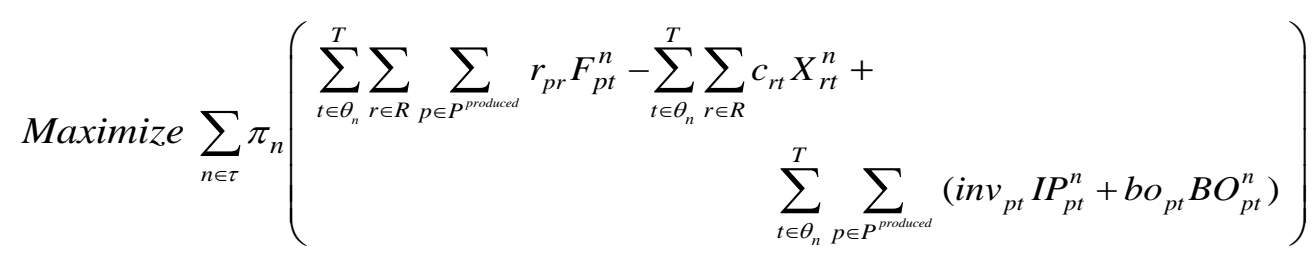

\section{B. Constraints}

$$
\begin{aligned}
& \sum_{\forall r \in R} \delta_{r} X_{r t}^{n} \leq c_{t} \\
& \forall t \in \theta_{n}, n \in \tau \\
& \begin{array}{lr}
I C_{p t}^{n}=I C_{p t-1}^{n^{\prime}}+s_{p t}-\sum_{\forall r \in R} \phi_{p r} X_{r t}^{n} & \forall p \\
n^{\prime} & \\
I P_{p t}^{n}-B O_{p t}^{n}=I P_{p t-1}^{n^{\prime}}-B O_{p t-1}^{n^{\prime}}+\sum_{\forall r \in R} \rho_{p r} X_{r t}^{n}(n)-d_{p t}^{n}
\end{array} \\
& \forall p \in p^{\text {produced }}, t \in \theta_{n}, n \& n^{\prime} \in \tau \\
& n^{\prime}=n \text { if } t-1 \in \theta_{n} \text {; otherwise } n^{\prime}=\lambda_{n} \\
& I P_{p t}^{n}-B O_{p t}^{n}=I P_{p t-1}^{n^{\prime}}-B O_{p t-1}^{n^{\prime}}+\sum_{\forall r \in R} \rho_{p r} X_{r t}^{n}(n)-F_{p t}^{n} \\
& \forall p \in p^{\text {produced }}, t \in \theta_{n}, n \& n^{\prime} \in \tau \\
& n^{\prime}=n \text { if } t-1 \in \theta_{n} \text {; otherwise } n^{\prime}=\lambda_{n} \\
& F_{p t}^{n} \leq d_{p t}^{n} \quad \forall p \in p^{\text {consumed }}, t \in \theta_{n}, n \in \tau \\
& X_{r t}^{n} \geq 0, I C_{p t}^{n} \geq 0 \quad \forall p \in p^{\text {consumed }}, t \in \theta_{n}, n \in \tau, r \in R \\
& I P_{p t}^{n} \geq 0, B O_{p t}^{n} \geq 0 \quad \forall p \in p^{\text {produced }}, t \in \theta_{n}, n \in \tau \\
& F_{p t}^{n} \geq 0, \quad \forall p \in p^{\text {produced }}, t \in \theta_{n}, n \in \tau
\end{aligned}
$$

Therefore, summary of alternative models defines accordingly. 
International Journal of Mathematical, Engineering and Management Sciences

Vol. 6 No. 2, 522-540, 2021

https://doi.org/10.33889/IJMEMS.2021.6.2.032

Model 1: The objective function is given by Equation (16)

Subject to: (19)-(21) and (24)-(25).

Model 2: The objective function is given by Equation (17)

Subject to: (19)-(21) and (24)-(25).

Model 3: The objective function is given by Equation (18)

Subject to: (19)-(20) and (22)-(26).

When applying the compact formulation, the decision variables $X_{r t}^{n}$ are set for each node of the tree of demand scenarios tree and a set of periods in each stage denoted by $\theta_{n}$. Therefore, the control variable of model (16)-(26) is production plan $X_{r t}^{n}$. The state variables are the inventory quantity variable $\left(I P_{p t}^{n}\right)$ together with the backorder quantity variable $\left(B O_{p t}^{n}\right)$. They are used to transfer ending values related to inventory and backorder from the previous stage to the first period of relevant nodes at the present stage.

\section{Case Study}

Computational experiments are presented in this section with the proposed model applied in a Canadian real-scale softwood remanufacturing mill, in order to investigate the quality of production plans suggested by the new stochastic model compared to those of deterministic nature and to a two-stage stochastic programming model. The impact of demand variability is studied, and we compute the value of multi-stage stochastic programming for this case study. The proposed predictive multi-stage stochastic models are also compared to a reactive re-planning approach with rolling-horizon. We determine the number of stages for which the multi-stage programs provide better planning than the re-planning approach. In the following sub-sections, all input data and the experimental results are presented.

\subsection{Input Data}

Data from the case under study including a total of 107 products are used with a planning horizon of 27 days, five business days per week, three shifts and 7.5 hours per shift. In total, we have 18 business days with 3 shifts, resulting in 54 periods.

Upon order arrival, demands are often served in a time window of two business days. Since no sample data were available for due dates, hence we employed a symmetric triangular distribution to randomly generate due dates of demand. Moreover, we use the historical data of the case to estimate the demand patterns in terms of quantity. The normal distribution $N(\mu, \sigma)$ is fitted for the demand data, where $\mu$ is the mean and $\sigma$ is the standard deviation (S.D.). In order to model demand evolution, four demand patterns (DP) are considered (identical mean, different standard deviations). In Table 1 , in node $n$ a demand of one single product is donated by $x_{n}$, while $x_{1}$ represents the root node's demand.

In the scenario trees, stages quantity varies from 2 to 4 , and the nodes in these trees vary from 4 to 40. As a result, for each random demand pattern, three scenario trees are considered. For each data point, we conduct thirty independent experiments for each data point, with 30 runs in average. In 
International Journal of Mathematical, Engineering and Management Sciences

Vol. 6 No. 2, 522-540, 2021

https://doi.org/10.33889/IJMEMS.2021.6.2.032

total, 1080 problems were generated and solved with CPLEX 12.6.1 and OPL 6.2, on an Intel® Core $^{\mathrm{TM}}$ i7-4700HQ processor, $2.40 \mathrm{GHz}, 12 \mathrm{~GB}$ of RAM, and Microsoft Windows.

Table 1. Demand distributions.

\begin{tabular}{ccc}
\hline Demand Pattern & Characteristic & Distribution \\
\hline DP1 & Constant mean, S.D. =5\% mean & $x_{n} \sim x_{1} N(\mu, 0.05 \mu)$ \\
DP2 & Constant mean, S.D. =10\% mean & $x_{n} \sim x_{1} N(\mu, 0.1 \mu)$ \\
DP3 & Constant mean, S.D. =20\% mean & $x_{n} \sim x_{1} N(\mu, 0.2 \mu)$ \\
DP4 & Constant mean, S.D. =30\% mean & $x_{n} \sim x_{1} N(\mu, 0.3 \mu)$ \\
\hline
\end{tabular}

\subsection{Experimental Results}

The solutions of abovementioned models for the four demand patterns are compared in Table 2 .

Table 2. Results comparison of production planning approaches.

\begin{tabular}{|c|c|c|c|c|c|c|c|c|c|c|}
\hline \multirow{2}{*}{ DP } & \multirow[b]{2}{*}{ PPA } & \multicolumn{3}{|c|}{ Model 1} & \multicolumn{3}{|c|}{ Model 2} & \multicolumn{3}{|l|}{ Model 3} \\
\hline & & $\begin{array}{c}\text { Exp. T. } \\
\text { Cos. } \\
(\$)\end{array}$ & $\begin{array}{c}\text { Exp. } \\
\text { Inv./Bo } \\
\text { Cos. (\$) }\end{array}$ & $\begin{array}{c}\text { CPU } \\
\text { time } \\
(\mathbf{S})\end{array}$ & $\begin{array}{c}\text { Exp. } \\
\text { Mar. } \\
\text { Con. (\$) }\end{array}$ & $\begin{array}{c}\text { Exp. } \\
\text { Inv./Bo } \\
\text { Cos. (\$) }\end{array}$ & $\begin{array}{c}\text { CPU } \\
\text { time } \\
\text { (S) }\end{array}$ & $\begin{array}{l}\text { Exp. } \\
\text { Pro. } \\
\text { (\$) }\end{array}$ & $\begin{array}{l}\text { Exp. } \\
\text { Inv./Bo } \\
\text { Cos. (\$) }\end{array}$ & $\begin{array}{c}\text { CP } \\
\text { U } \\
\text { tim } \\
\mathbf{e} \\
(\mathbf{S})\end{array}$ \\
\hline Real & $\begin{array}{l}\text { Re- } \\
\text { planning }\end{array}$ & 635,826 & 91,023 & 35 & 74,689 & 53,380 & 35 & 78,929 & 89,564 & 35 \\
\hline \multirow{4}{*}{ DP1 } & 4-Stage SP & 632,365 & 46,894 & 824 & 78,411 & 51,862 & 824 & $\begin{array}{c}111,58 \\
1\end{array}$ & 48,726 & 824 \\
\hline & 3-Stage SP & 633,668 & 47,255 & 252 & 74,301 & 54,316 & 252 & 82,510 & 49,044 & 252 \\
\hline & 2-Stage SP & 653,181 & 47,641 & 93 & 64,174 & 56,221 & 93 & 57,840 & 51,033 & 93 \\
\hline & $\begin{array}{c}\text { Determinis } \\
\text { tic }\end{array}$ & 684,929 & 51,193 & 0 & 46,852 & 67,822 & 0 & 35,340 & 52,574 & 0 \\
\hline \multirow{4}{*}{ DP2 } & 4-Stage SP & 633,301 & 49,058 & 825 & 76,312 & 52,558 & 825 & 99,613 & 49,058 & 825 \\
\hline & 3-Stage SP & 638,132 & 51,069 & 250 & 73,755 & 54,747 & 250 & 76,448 & 51,136 & 250 \\
\hline & 2-Stage SP & 655,388 & 52,041 & 91 & 64,063 & 57,492 & 91 & 50,826 & 52,175 & 91 \\
\hline & $\begin{array}{c}\text { Determinis } \\
\text { tic }\end{array}$ & 686,828 & 53,030 & 0 & 40,215 & 73,819 & 0 & 32,660 & 52,988 & 0 \\
\hline \multirow{4}{*}{ DP3 } & 4-Stage SP & 635,120 & 61,108 & 826 & 74,789 & 53,264 & 826 & 91,075 & 51,800 & 826 \\
\hline & 3-Stage SP & 639,787 & 61,404 & 254 & 73,175 & 55,106 & 254 & 71,981 & 52,104 & 254 \\
\hline & 2-Stage SP & 660,420 & 62,882 & 92 & 63,539 & 57,737 & 92 & 48,494 & 52,935 & 92 \\
\hline & $\begin{array}{c}\text { Determinis } \\
\text { tic }\end{array}$ & 695,417 & 68,227 & 0 & 35,353 & 79,758 & 0 & 29,588 & 53,494 & 0 \\
\hline \multirow{4}{*}{ DP4 } & 4-Stage SP & 638,928 & 72,432 & 823 & 73,897 & 54,243 & 823 & 82,257 & 67,404 & 823 \\
\hline & 3-Stage SP & 642,728 & 73,066 & 251 & 71,887 & 55,619 & 251 & 64,843 & 73,060 & 251 \\
\hline & 2-Stage SP & 667,227 & 80,132 & 93 & 63,397 & 58,055 & 93 & 44,879 & 80,132 & 93 \\
\hline & $\begin{array}{l}\text { Determinis } \\
\text { tic }\end{array}$ & 697,314 & 80,888 & 0 & 32,587 & 81,652 & 0 & 27,985 & 84,938 & 0 \\
\hline
\end{tabular}


In Table 2, "DP" represents the demand pattern, "PPA" represents the production planning approach, "Exp. T. Cos." represents the expected total cost, "Exp. Inv./Bo. Cos." represents the expected Inventory/backorder cost, "Exp. Mar. Con." represents the expected marginal contribution, "Exp. Pro." represents the expected profit, and the SP is the stochastic programming. Several observations can be made regarding the nature of the optimal solutions for the different models of Table 2 .

Observation 1. Impact of Higher Demand Variability

The first observation from Table 2 is that when the demand variability is higher (from DP1 to DP4), the expected cost becomes bigger in similar planning approaches; whereas the marginal contribution and profit of these models become smaller. As an example, the cost of 4-stage SP models goes up from DP1 to DP4, whereas the profit of 4-stage SP models goes down from DP1 to DP4. This is valid for all similar planning approaches. Thus, the multi-stage stochastic models exhibit higher quality solutions when the demand variability increases.

Figures 1, Figure 2 and Figure 3 indicate that the expected cost of plans in Model 1 becomes bigger for higher demand variability. For instance, this difference in DP3 between 4-stage SP and 2-stage $\mathrm{SP}$ is $\$ 25,300$, whereas this difference in DP4 is $\$ 28,299$ (see Table 2). In contrast, this difference would be smaller in Models 2 and 3 due to their objective functions perspective. For instance, this difference in DP3 of Model 2 between 4-stage SP and 2-stage SP is $\$ 11,250$, whereas this difference in DP4 is $\$ 10,500$. In both situations, this shows the importance of using the multi-stage stochastic models rather than two-stage or deterministic models in this situation. When demand variability is higher, the objective function deteriorates; and higher number of stages leads to better solutions.

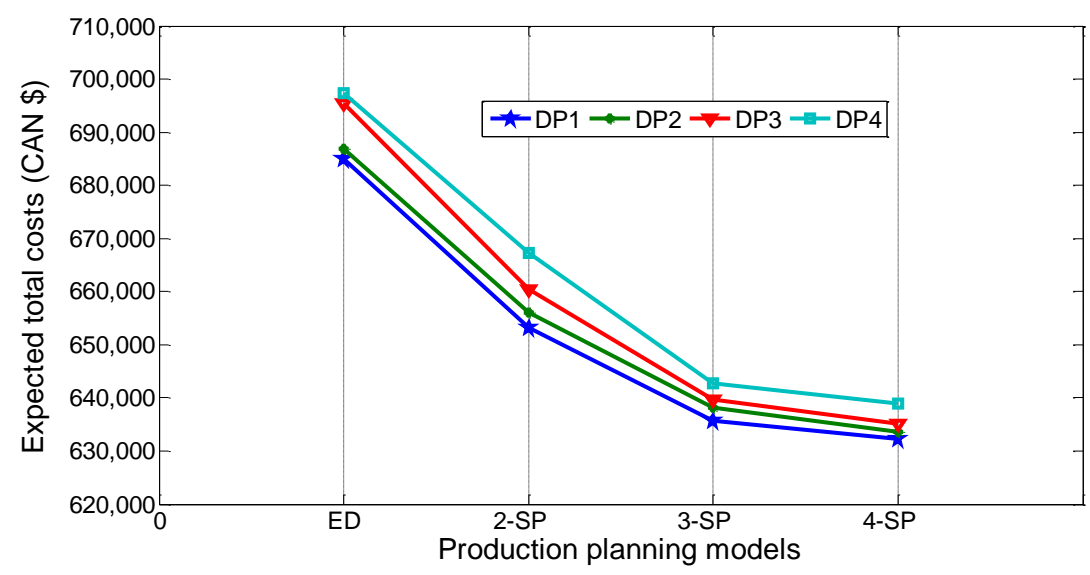

Figure 1. Expected total costs (Model 1). 
International Journal of Mathematical, Engineering and Management Sciences

Vol. 6 No. 2, 522-540, 2021

https://doi.org/10.33889/IJMEMS.2021.6.2.032

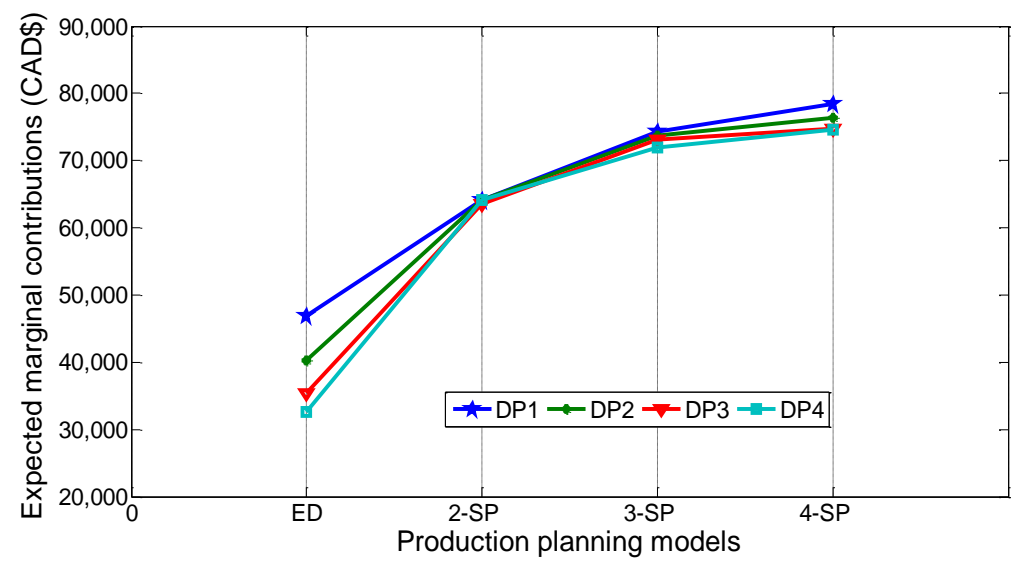

Figure 2. Expected marginal contributions (Model 2).

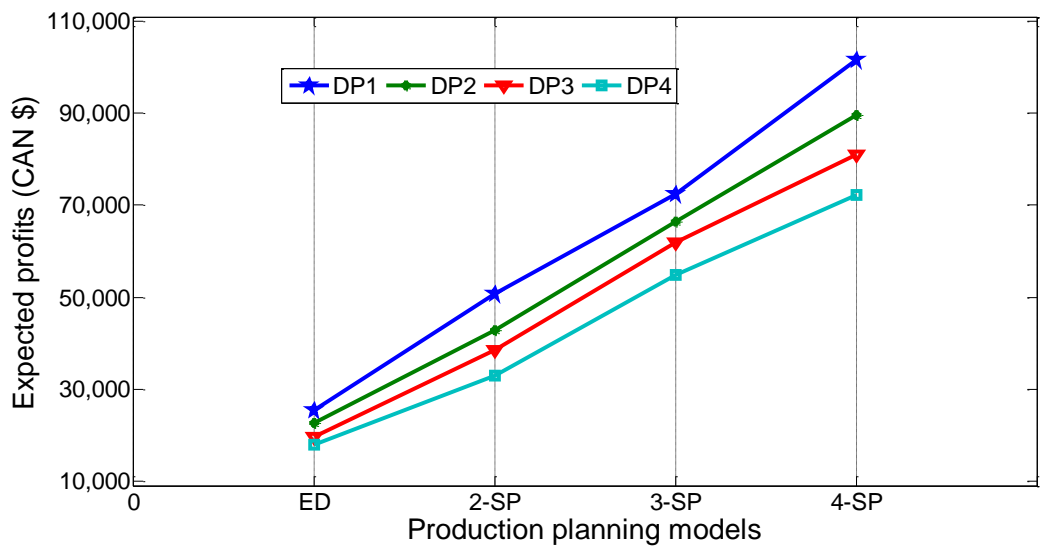

Figure 3. Expected profits (Model 3).

To better understand the effect of demand variations on optimization models, the Coefficient of Variance $(\mathrm{CV})$ of different demand patterns is calculated in Table 3.

Table 3. CV of planning approaches for optimal solutions of Models 1-3.

\begin{tabular}{cccc}
\hline Planning Approach & $\begin{array}{c}\text { Model 1 } \\
\text { (Min. Cost) }\end{array}$ & $\begin{array}{c}\text { Model 2 } \\
\text { (Max. Marginal Contribution) }\end{array}$ & $\begin{array}{c}\text { Model 3 } \\
\text { (Max. Profit) }\end{array}$ \\
\hline 4-Stage SP & 0.00 & 0.03 & 0.13 \\
3-Stage SP & 0.01 & 0.01 & 0.13 \\
2-Stage SP & 0.01 & 0.01 & 0.11 \\
\hline
\end{tabular}

In Table 3, the CV of planning approaches in Models 1 and 2 has small values. In contrast, these values for Model 3 are slightly higher. From statistics, we know that the higher the CV, the greater the dispersion in the model. As a result, the effect of demand pattern variation on Model 3 is more important than other models. 
Observation 2. Impact on the Expected Inventory/Backorder Cost

To show the impact of the proposed approaches on the expected inventory/backorder cost, a full factorial experimental design is done, considering the number of stages and demand pattern as input factors affecting responses. Three-dimensional response-surface plots are drawn. Figure 4(a), Figures 4(b) and Figure 4(c) present the response-surface plots for inventory/backorder costs as a function of number of stages and demand pattern. In these figures, a linear function of the factors creates the expected response. In fact, the $R(t, u)$ is the response for a specific pair $(t, u)$ that takes the following form:

$$
E[R(t, u)]=\beta_{0}+\beta_{t} t+\beta_{u} u+\beta_{t u} t u .
$$

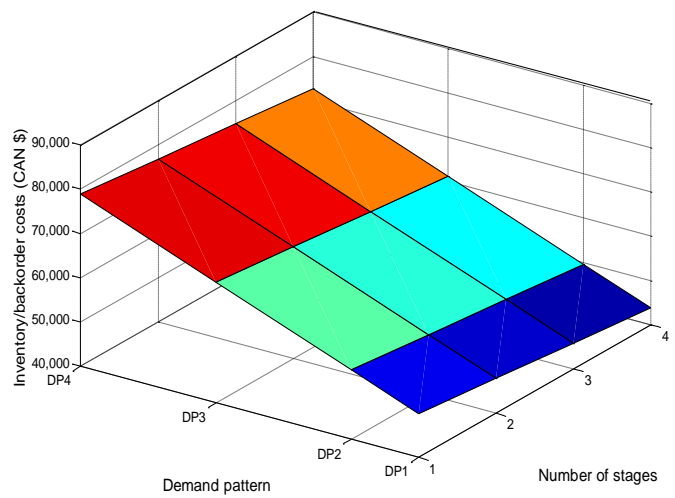

Figure 4(a)

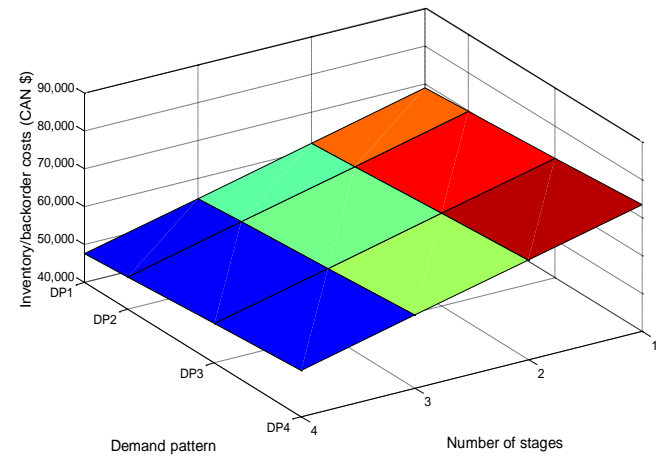

Figure 4(b)

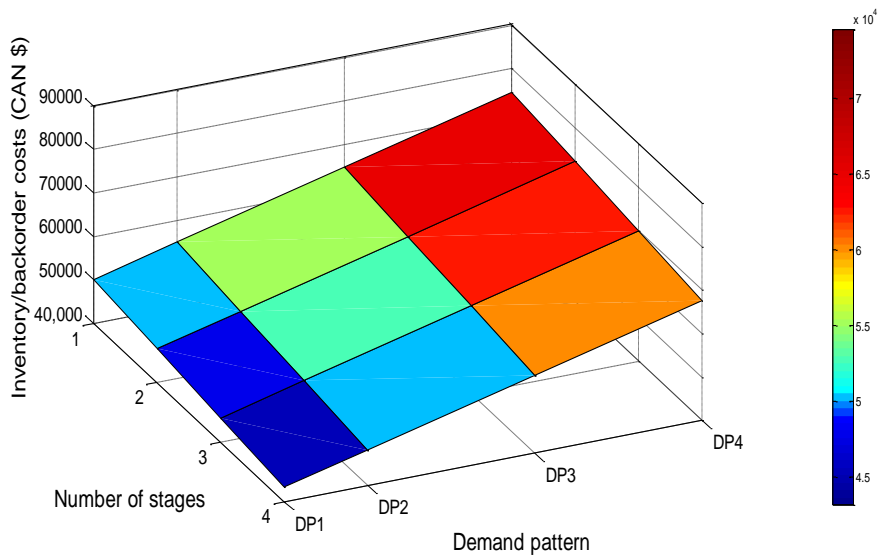

Figure 4(c)

Figure 4. Expected inventory/backorder costs respectively in Models 1, 2 and 3.

In Figure 4(a), start from factor DP1 and go on the number of stages axis towards stage 4. As the number of stages increases, the inventory/backorder cost decreases. This observation is valid for other DPs in this figure. In Figure 4(b) and Figure 4(c), costs decline when increasing the number 
International Journal of Mathematical, Engineering and Management Sciences

Vol. 6 No. 2, 522-540, 2021

https://doi.org/10.33889/IJMEMS.2021.6.2.032

of stages, the inventory/backorder. In addition, these figures suggest that the rise in demand variability causes an increase of the inventory/backorder costs in each stage.

Observation 3. Impact of the CPU Time

The CPU time suggest that the high quality of multi-stage models needs larger computational time paralleled to those of the re-planning (RP), two-stage (2-stage) SP and deterministic ones. Despite the high computational (but reasonable) time of the multi-stage models in comparison with other models, these models provide high quality solutions according to the following observation.

Observation 4. Quality of Multi-Stage SP Solutions versus RP Solutions

In the re-planning optimization coupled with rolling horizon approach, we have to take into consideration the re-planning period length and the planning horizon. In this case, a plan is divided into different static plans organized at regular intervals. In addition, plans are not recalculated until the next interval (c.f., Rafiei et al., 2014). Various combinations of these two factors can be taken into account, but we have chosen a re-planning period length 3 (i.e. revising the plan every 3 periods - 1 day) and a level of planning horizon of 27 periods (denoted by RP (3-27)) as the best setting for this case according to Rafiei et al. (2014). Besides this setting, we use two other sets; RP (6-27) and RP (9-27), which have 6 periods ( 2 days) and 9 periods (3 days) respectively as the re-planning intervals with the same 27-period rolling horizon.

In the 4-stage SP approach, the 54-period planning horizon is organized in 3 stages with 18 periods (6 days) each. Similarly, the 3-stage SP approach employed a planning horizon with 54 periods organized in 2 stages with 27 periods each. Table 4 shows the relative difference of solutions in multi-stage stochastic programming and re-planning approaches.

Table 4. Relative difference of solutions in multi-stage SP and re-planning.

\begin{tabular}{|c|c|c|c|c|c|c|c|c|c|}
\hline \multirow{2}{*}{$\begin{array}{l}\text { Multi- } \\
\text { stage } \\
\text { model }\end{array}$} & \multicolumn{3}{|c|}{$\begin{array}{c}\text { Model } 1 \\
\text { (Min. Cost) }\end{array}$} & \multicolumn{3}{|c|}{$\begin{array}{c}\text { Model 2 } \\
\text { (Max. Marginal Contribution) }\end{array}$} & \multicolumn{3}{|c|}{$\begin{array}{c}\text { Model } 3 \\
\text { (Max. Profit) }\end{array}$} \\
\hline & $\begin{array}{c}\mathrm{RP} \\
(3-27)\end{array}$ & $\begin{array}{c}\mathrm{RP} \\
(6-27)\end{array}$ & $\begin{array}{c}\mathrm{RP} \\
(9-27)\end{array}$ & $\begin{array}{c}\mathrm{RP} \\
(3-27)\end{array}$ & $\begin{array}{c}\mathrm{RP} \\
(6-27)\end{array}$ & $\begin{array}{c}\mathrm{RP} \\
(9-27)\end{array}$ & $\begin{array}{c}\mathrm{RP} \\
(3-27)\end{array}$ & $\begin{array}{c}\mathrm{RP} \\
(6-27)\end{array}$ & $\begin{array}{c}\mathrm{RP} \\
(9-27)\end{array}$ \\
\hline $\begin{array}{l}\text { 4-stage } \\
\text { SP }\end{array}$ & $-1.1 \%$ & $-10.1 \%$ & $-17.9 \%$ & $3.2 \%$ & $34.3 \%$ & $71.2 \%$ & $21.8 \%$ & $101.5 \%$ & $219.3 \%$ \\
\hline $\begin{array}{c}\text { 3-stage } \\
\text { SP }\end{array}$ & $0.2 \%$ & $-8.7 \%$ & $-16.8 \%$ & $-1.9 \%$ & $26.3 \%$ & $62.8 \%$ & $-6.3 \%$ & $87.9 \%$ & $145.6 \%$ \\
\hline
\end{tabular}

By being sensitive to the objective function and considering relative difference in Table 4, the 4stage SP decreases the costs in Model 1 by $1.1 \%, 10.1 \%$, and $17.9 \%$ respectively in comparison with the cost values in RP (3-27), RP (6-27), and RP (9-27). In Model 2, the marginal contribution in 4-stage SP has a $3.2 \%, 34.3 \%$, and $71.2 \%$ increase respectively in comparison with the marginal contribution in RP (3-27), RP (6-27), and RP (9-27). In Model 3, the profit of 4-stage SP is 21.8\%, $101.5 \%$, and $219.3 \%$ more respectively in comparison with the profit in RP (3-27), RP (6-27), and RP (9-27). Accordingly, the solutions of 4-stage SP in all models are better than the RP solutions and they are even better than the best re-planning setting solutions (RP (3-27)). However, this is not valid for the 3-stage SP. The quality of 3-stage SP solutions is slightly less than RP (3-27) solutions in all models. However, the solutions of RP (6-27) and RP (9-27) are far inferior to 3stage SP solutions in all models. As a conclusion, even the solutions of the best considered replanning setting fall behind the 4-stage stochastic programming solutions. 
International Journal of Mathematical, Engineering and Management Sciences

Vol. 6 No. 2, 522-540, 2021

https://doi.org/10.33889/IJMEMS.2021.6.2.032

Observation 5. Value of the Proposed Model

The proposed models' value were evaluated using the "Value of Multi-Stage Stochastic Programming" (VMS) proposed by Huang and Ahmed (2009). VMS captures the benefit received of solving the stochastic recourse problem versus expected value of the deterministic problem. Note that here VMS is adapted according to the objective functions perspective. For minimization objectives, the VMS percentage is defined as: $\left(\frac{v^{T S}-v^{M S}}{v^{M S}}\right) * 100$ where $v^{T S}$ and $v^{M S}$ are respectively the optimum objective values of 2-stage SP and 4-stage SP. In maximization objectives, the VMS percentage is defined as $\left(\frac{v^{M S}-v^{T S}}{v^{T S}}\right) * 100$. The differences indicate how much savings, marginal contribution, or profit can be realized by using 4-stage SP as against 2stage SP depending on the optimization perspective. Table 5 shows that in all the DPs, the solutions of the multi-stage programming are far superior to the two-stage models with respect to the relevant objective function. It also demonstrates that the magnitude of the VMS is influenced by the pattern of the demand distribution.

Table 5. Percentage value of multi-stage stochastic programming.

\begin{tabular}{cccc}
\hline $\begin{array}{c}\text { Demand } \\
\text { pattern }\end{array}$ & $\begin{array}{c}\text { Model 1 } \\
\text { (Min. Cost) }\end{array}$ & $\begin{array}{c}\text { Model 2 } \\
\text { (Max. Marginal Contribution) }\end{array}$ & $\begin{array}{c}\text { Model 3 } \\
\text { (Max. Profit) }\end{array}$ \\
\hline DP1 & $3.3 \%$ & $22.2 \%$ & $92.9 \%$ \\
DP2 & $3.5 \%$ & $19.1 \%$ & $96.0 \%$ \\
DP3 & $4.0 \%$ & $17.7 \%$ & $87.8 \%$ \\
DP4 & $4.4 \%$ & $16.6 \%$ & $83.3 \%$ \\
\hline
\end{tabular}

\section{Summary of Observations}

This research proposed and compared novel multi-stage stochastic models to the reactive approach, as well as to determine the number of stages for which the multi-stage programs provide better planning than the re-planning approach. In that regard, we observe that, although the daily-based re-planning (RP (3-27)) approach has reasonable results in the reality of the case studied, the high level of re-planning frequency (daily basis) makes it harder for planners. The resulting short replanning intervals increase the number of revisions that a plan undergoes during execution; whereas the multi-stage models allow for fewer revisions (6 days as a stage) creating more stability in plans and improving nervousness. An insight from the abovementioned analysis is that the re-planning approach as the current practice of the case under study can be improved by applying multi-stage stochastic programming. Indeed, the obtained solutions of the multi-stage model successfully outperform in competition with the re-planning approach. Among the multi-stage approaches, the 4-stage is chosen because the 3-stage SP model is not good enough in comparison with the best set of RP approaches (RP (3-27)). In fact, the 4-stage SP model provides much better results than the RP approaches along with a manageable time. Moreover, although the CPU time of the 4-stage SP model is much higher than others, it still has reasonable processing time. We also observe that when demand becomes more volatile, the 4-stage SP model performs much better than the others. Another observation is that by increasing the number of stages from three to four, the difference between the expected values of the objective functions of both 3-stage and 4-stage programming may not be important in the all models (see Figure 1, Figure 2 and Figure 3). Hence, it was not necessary to include more stages in the test problems' scenario trees. Furthermore, the 4-stage SP's quality is far greater than the one of the 2-stage SP. 
International Journal of Mathematical, Engineering and Management Sciences

Vol. 6 No. 2, 522-540, 2021

https://doi.org/10.33889/IJMEMS.2021.6.2.032

\section{Conclusion}

This article developed three new multi-stage stochastic programming models to deal with the challenging characteristics of the production planning process of wood remanufacturing industry. The proposed models were then compared using a dataset from a Canadian wood remanufacturing mill. Numerical experiments suggest that the 4-stage stochastic model performs better than the reactive planning model. In particular, results of different demand patterns proved the utility of the multi-stage SP when demand variations increase. The proposed approach determines the number of stages for which the multi-stage programs provide better planning than the re-planning approach. This allows for an evaluation of the gain of using a predictive stochastic programming model, instead of a reactive planning approach that may increase the system nervousness and instability. All the observed results are conforming to the spirit of stochastic programming approaches. These results demonstrate that the proposed models are valid and can be advantageously used by our industrial partner and other wood remanufacturing companies as well.

As a further extension of this study, a robust optimization approach can be employed to explicitly deal with demand variability. Incorporating the raw material and/or price unpredictability may be an interesting research avenue. In this case, new approaches will be required to tame the intricacy of the stochastic planning model, increasing the chances it will be adopted in practice.

\section{Conflict of Interest}

The authors confirm that there is no conflict of interest to declare for this publication.

\section{Acknowledgements}

Authors express their sincere thanks to industrial partner operating in Canada.

\section{References}

Alfieri, A., \& Brandimarte, P. (2005). Stochastic programming models for manufacturing applications. In: Matta, A., Semeraro, Q. (eds) Design of Advanced Manufacturing Systems. Springer, Dordrecht, pp. 73-124.

Alonso-Ayuso, A., Escudero, L.F., \& Ortuno, M.T. (2003). BFC, A branch-and-fix coordination algorithmic framework for solving some types of stochastic pure and mixed 0-1 programs. European Journal of Operational Research, 151(3), 503-519.

Birge, J.R., \& Louveaux, F. (2010). Introduction to stochastic programming. Second edition, Springer, New York.

Brandimarte, P. (2006). Multi-item capacitated lot-sizing with demand uncertainty. International Journal of Production Research, 44(15), 2997-3022.

Chu, Y., \& You, F. (2013). Integration of scheduling and dynamic optimization of batch processes under uncertainty: two-stage stochastic programming approach and enhanced generalized benders decomposition algorithm. Industrial \& Engineering Chemistry Research, 52(47), 16851-16869.

D'amours, S., Rönnqvist, M., \& Weintraub, A. (2008). Using operational research for supply chain planning in the forest products industry. INFOR: Information Systems and Operational Research, 46(4), 265-281.

Engell, S., Märkert, A., Sand, G., \& Schultz, R. (2004). Aggregated scheduling of a multiproduct batch plant by two-stage stochastic integer programming. Optimization and Engineering, 5(3), 335-359. 
International Journal of Mathematical, Engineering and Management Sciences

Vol. 6 No. 2, 522-540, 2021

https://doi.org/10.33889/IJMEMS.2021.6.2.032

Farrell, R.R., \& Maness, T.C. (2005). A relational database approach to a linear programming-based decision support system for production planning in secondary wood product manufacturing. Decision Support Systems, 40(2), 183-196.

Gupta, A., \& Maranas, C.D. (2003). Managing demand uncertainty in supply chain planning. Computers \& Chemical Engineering, 27(8-9), 1219-1227.

Higle, J.L. (2005). Stochastic programming: optimization when uncertainty matters. In: Greenberg, H.J., Smith, J.C. (eds.) INFORMS TutORials in Operations Research. INFORMS, Hanover, pp. 30-53

Huang, K., \& Ahmed, S. (2009). The value of multistage stochastic programming in capacity planning under uncertainty. Operations Research, 57(4), 893-904.

Karabuk, S., \& Wu, S.D. (2003). Coordinating strategic capacity planning in the semiconductor industry. Operations Research, 51(6), 839-849.

Kazemi, Z.M., Nourelfath, M., \& Ait-Kadi, D. (2010). A multi-stage stochastic programming approach for production planning with uncertainty in the quality of raw materials and demand. International Journal of Production Research, 48(16), 4701-4723.

Kazemi, Z.M., Nourelfath, M., \& Ait-Kadi, D. (2011). Production planning with uncertainty in the quality of raw materials. Journal of Operation Research Society, 62(7), 1334-1343.

Kozak, R.A., Maness, T.C., \& Caldecott, T. (2003). Solid wood supply impediments for secondary wood producers in British Columbia. The Forestry Chronicle, 79(6), 1107-1120.

Miller III, A.C., \& Rice, T.R. (1983). Discrete approximations of probability distributions. Management Science, 29(3), 352-362.

Pimentel, B.S., Mateus, G.R., \& Almeida, F.A. (2013). Stochastic capacity planning and dynamic network design. International Journal of Production Economics, 145(1), 139-149.

Pirraglia, A., Saloni, D., \& Van Dyk, H. (2009). Status of lean manufacturing implementation on secondary wood industries including residential, cabinet, millwork, and panel markets. BioResources, 4(4), 13411358.

Rafiei, R., Nourelfath, M., Gaudreault, J., De Santa-Eulalia, L.A., \& Bouchard, M. (2016). Analysis of uncontrollable supply effects on a co-production demand-driven wood remanufacturing mill with alternative processes. INFOR: Information Systems and Operational Research, 54(3), 234-261.

Rafiei, R., Nourelfath, M., Gaudreault, J., De Santa-Eulalia, L.A., \& Bouchard, M. (2015). Dynamic safety stock in co-production demand-driven wood remanufacturing mills: a case study. International Journal of Production Economics, 165, 90-99.

Rafiei, R., Nourelfath, M., Gaudreault, J., Santa-Eulalia, L.A., \& Bouchard, M. (2014). A periodic replanning approach for demand-driven wood remanufacturing industry: a real-scale application. International Journal of Production Research, 52(14), 4198-4215.

Rao, U.S., Swaminathan, J.M., \& Zhang, J. (2004). Multi-product inventory planning with downward substitution, stochastic demand and setup costs. IIE Transactions, 36(1), 59-71.

Rönnqvist, M., D’Amours, S., Weintraub, A., Jofre, A., Gunn, E., Haight, R.G., Martell, D., Murray, A.T., \& Romero, C. (2015). Operations research challenges in forestry: 33 open problems. Annals of Operations Research, 232(1), 11-40.

Ruszczynski, A., \& Shapiro, A. (2003). Stochastic programming. Handbooks in Operations Research and Management Science, 10, 1-64.

Shabani, N., Sowlati, T., Ouhimmou, M., \& Rönnqvist, M. (2014). Tactical supply chain planning for a forest biomass power plant under supply uncertainty. Energy, 78, 346-355. 
International Journal of Mathematical, Engineering and Management Sciences

Vol. 6 No. 2, 522-540, 2021

https://doi.org/10.33889/IJMEMS.2021.6.2.032

Swaminathan, J.M. (2000). Tool capacity planning for semiconductor fabrication facilities under demand uncertainty. European Journal of Operational Research, 120(3), 545-558.

Vieira, G.E., Herrmann, J.W., \& Lin, E. (2003). Rescheduling manufacturing systems: a framework of strategies, policies, and methods. Journal of Scheduling, 6(1), 39-62.

(c) (i)

Original content of this work is copyright @ International Journal of Mathematical, Engineering and Management Sciences. Uses under the Creative Commons Attribution 4.0 International (CC BY 4.0) license at https://creativecommons.org/licenses/by/4.0/ 